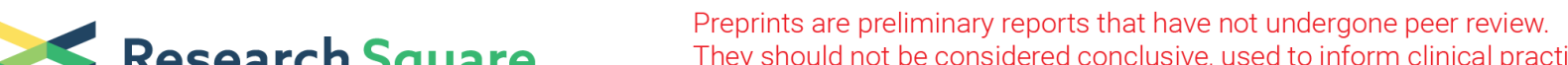 $\begin{array}{ll}\text { Research Square } & \text { They should not be considered conclusive, used to inform clinical practice, } \\ \text { or referenced by the media as validated information. }\end{array}$
}

\section{miR-138-5p Inhibits Vascular Mimicry by Targeting the HIF-1a/VEGFA Pathway in Hepatocellular Carcinoma}

\section{Hongwei Liu}

The Sixth Affiliated Hospital of Guangzhou Medical University

\section{Xiujin Hu}

The Sixth Affiliated Hospital of Guangzhou Medical University

\section{Weihe Tan}

The Sixth Affiliated Hospital of Guangzhou Medical University

\section{Peng Zhou}

The Sixth Affiliated Hospital of Guangzhou Medical University

\section{Yanmei Liu}

The Sixth Affiliated Hospital of Guangzhou Medical University

\section{Chen Chen}

The Sixth Affiliated Hospital of Guangzhou Medical University

\section{Meng Yang}

The Sixth Affiliated Hospital of Guangzhou Medical University

\section{Meifang Zhou}

The Sixth Affiliated Hospital of Guangzhou Medical University

\section{Shuxia Xuan}

The Sixth Affiliated Hospital of Guangzhou Medical University

\section{Bing Cheng}

The Sixth Affiliated Hospital of Guangzhou Medical University

\section{Weiguo Yin}

The Sixth Affiliated Hospital of Guangzhou Medical University

Jinduan Lin ( $2019695160 @ g z h m u . e d u . c n$ )

The Sixth Affiliated Hospital of Guangzhou Medical University

\section{Research Article}

Keywords: miR-138-5p, vascular mimicry, hypoxia-inducible factor $1 \mathrm{a}$, vascular endothelial growth factor A

Posted Date: December 22nd, 2021 
DOI: https://doi.org/10.21203/rs.3.rs-1080163/v1

License: (c) (1) This work is licensed under a Creative Commons Attribution 4.0 International License. Read Full License 


\section{Abstract}

Tumor vascular mimicry (VM) is the process of new blood vessels formed by tumor cells rather than endothelial cells. An increasing number of researches have revealed that VM process is associated with cancer progression and metastasis. miR-138-5p has been reported to act as a tumor suppressor in many cancers. However, the role and underlying mechanism of miR-138-5p in hepatocellular carcinoma (HCC) VM remain unclear. In this study, VM density was detected by CD31/periodic acid-Schiff double staining in HCC clinical specimens. We found that miR-138-5p expression correlated strongly negatively with microvessel density. Additionally, miR-138-5p mimic or inhibitor decreased or increased, respectively, tube formation capacity in HepG2 and Hep3B cells. Consistent with this, miR-138-5p repressed vessel density in vivo. Moreover, miR-138-5p targeted hypoxia-inducible factor $1 \mathrm{a}(\mathrm{HIF-1a)}$ and regulated expression of HIF-1a and vascular endothelial growth factor A (VEGFA), which are established classical markers of angiogenesis. Consistent with these findings, the HIF-1 a inhibitor CAY10585 effectively blocked HCC cell VM and VEGFA expression. In conclusion, miR-138-5p inhibits HepG2 and Hep3B cell VM by blocking the HIF-1a/VEGFA pathway. Therefore, miR-138-5p may serve as a useful therapeutic target for miRNA-based HCC therapy.

\section{Introduction}

Hepatocellular carcinoma (HCC) is the most common primary liver malignancy and is the top third cause of cancer-related death worldwide ${ }^{[1,2]}$. Accumulated evidence indicates that vascular invasion and metastasis play important roles in the high mortality rate of patients with $\mathrm{HCC}^{[3,4]}$. Tumor growth and metastasis require an adequate blood supply to transport nutrients and oxygen and remove metabolic waste, providing a pathway for tumor metastasis and stimulating growth of the tumor mass. Along with the endothelial vessels (EVs), tumor vascular mimicry (VM) occurs via the generation of a de novo vascular network by tumor cells to supply nutrition for sustaining tumor growth. EV and VM are both important processes in the tumorigenicity and metastasis of HCC.

MicroRNAs (miRNAs) are one kind of noncoding RNAs with approximately 22 nucleotides in length that have an important function in many essential physiological and pathological processes. miRNAs regulate target genes at posttranscriptional level by binding to their $3^{\prime}-\mathrm{UTR}^{[5,6]}$. It has been reported that miR-138$5 p$ acts as a tumor suppressor to inhibit tumor growth and metastasis in vivo and in vitro ${ }^{[7-9]}$. Moreover, recent reports have demonstrated that miR-138-5p inhibits endothelial progenitor cell proliferation by inhibiting hypoxia-inducible factor $1 a(H I F-1 a)^{[10,11]}$. HIF-1 $a$ is an important molecule for EVs and VM. Given that miR-138-5p affects both tumor cells and endothelial cells and regulates the expression level of HIF-1a in endothelial cells, we hypothesized that miR-138-5p might suppress tumor growth by blocking blood nutrient supply. The effects of miR-138-5p on VM have not been reported in any type of cancer. In this study, we found that miR-138-5p inhibited VM and regulated HIF-1a/vascular endothelial growth factor A (VEGFA) expression by targeting HIF-1a in HCC cells. 


\section{Materials And Methods}

\section{Tissue specimens}

Twenty-three pairs of fresh human HCC and corresponding paratumor tissues were obtained from surgical specimens immediately after their resection from patients who underwent routine surgery at the Qingyuan Hospital, The Six Affiliated Hospital of Guangzhou Medical University (Qingyuan, China). No patient received preoperative irradiation or chemotherapy. The research protocol was approved by the Ethics Committee of The Six Affiliated Hospital of Guangzhou Medical University (No. QPH-IRBA0153). All experiments were carried out in accordance with the international ethical guidelines for biomedical research involving human subjects.

\section{Antibodies and an inhibitor}

Antibodies against HIF-1 $a$ antibody and VEGFA antibody were respectively purchase from Abcam and ABclonal Company for western blotting and immunohistochemistry (IHC). CAY10585 (Abcam) was used to inhibit HIF-1a.

\section{Cell culture}

Human HCC cell lines included the HepG2 (hepatoblastoma cell line), Hep3B, MHCC97H, MHCC97L, Huh7, SK-Hep1, and MIHA were purchase from the Chinese Academy of Sciences. MIHA human immortalized normal hepatocytes were used for the normal control cells. The cells were cultured in Dulbecco's modified Eagle's medium (Gibco) supplemented with $10 \%$ fetal bovine serum (Gibco) and $1 \%$ penicillin-streptomycin (Gibco) and incubated at $37^{\circ} \mathrm{C}$ in a humidified atmosphere containing $5 \% \mathrm{CO}_{2}$. In hypoxic condition, cells were cultured in the atmosphere of $1 \% 02$.

\section{RNA extra and quantitative real-time polymerase chain reaction (qRT-PCR)}

Total RNA was obtain from cultured cells or human tissues by using TRIzol reagent (Takara). qRT-PCR assay was conducted to deternmin the mRNA levels by using SYBR Green PCR Master Mix kit (Takara). $\beta$ Actin was used as an internal control to analyze HIF-1a and VEGFA mRNA levels. U6 expression was used as an internal control for analyzing miR-138-5p level in HCC tissue and HCC cell lines. $2^{-\Delta \triangle C T}$ method was used to assess the relative level of HIF-1 $a$ and VEGFA mRNA levels. All assays were evaluated in at least three repeats, all the results are shown as the mean \pm standard deviation (SD) for analysis. The primers are shown in Supplemental Table 1. The median expression level was used as the cutoff. Low expression was classified as values below the 50th percentile. High miR-138-5p expression was classified as values at or above the 50th percentile.

\section{RNA interference and transfection}

Hep3B and HepG2 cells were seeded into a 6-well plate at 30-50\% confluence. $24 \mathrm{~h}$ later, the cells were transfected with the HIF-1a inhibitor CAY10585 (Abcam) or transfected with a miR-138-5p mimic (Sangon 
Biotech) or a miR-138-5p inhibitor (Sangon Biotech) using the Lipofectamine 2000 reagent (Invitrogen) according to the manufacturer's instructions. Cells transfected with a scramble control siRNA (NC) were used as controls. The cells were harvested $48 \mathrm{~h}$ after the transfection. The sequences of the siRNA against HIF-1a, miR-138-5p mimic and miR-138-5p inhibitor are listed in Supplemental Table 1.

\section{Luciferase assay}

To test the direct binding of miR-138-5p to the target gene, HIF-1a, a luciferase reporter assay was performed as previously described. Luciferase activity was measured $48 \mathrm{~h}$ posttransfection using a DualLuciferase assay kit (Promega).

\section{Immunohistochemistry}

We used IHC assay by an IHC kit (Boster Bio-Engineering Company) to detect protein levels of VEGFA and HIF-1a. We also used CD31/periodic acid-Schiff (PAS) double staining to detect VM density. The staining intensity was represented by scores as follows: 0 -negative, 1-weak, 2-medium, and 3-strong. The staining extent scores were as bellows: 0 represents for $<10 \%$ ), 1 represents for $11-25 \%, 2$ represents for $26-50 \%, 3$ represents for $51-75 \%$, and 4 represents for $76-100 \%$. The final protein expression score was calculated as the intensity score $\times$ extent score and ranged from 0 to 12 .

\section{Protein extraction and western blotting}

Protein extraction kits (Keygen Biotech) were used to extract proteins from HepG2 and Hep3B cells. The protein concentrations were determined by a bicinchoninic acid protein assay kit (Keygen Biotech). WB was conducted according to the literature. Quantification was performed using the ImageJ software.

\section{Bioinformatics analysis}

The TargetScan 3.1 online software (http://www.targetscan.org) was used to predict the putative target genes of miR-138-5p. The species was "human," and "miR-138-5p" was entered as the miRNA name.

\section{Tube formation assay}

We choose the HepG2 and Hep3B cell line to carry out the gain or loss of function experiments according to the basic expression level of miR-138-5p and the basic tube formation ability. We used Matrigel (BD Biosciences) to perform the tube formation assay. After $50 \mu \mathrm{l}$ Matrigel polymerizationin a 96-well plate ( $37^{\circ} \mathrm{C}, 30 \mathrm{~min}$ ), 30,000 HepG2 and Hep3B cells were added onto the Matrigel each well. After $6 \mathrm{~h}$ incubation $\left(37^{\circ} \mathrm{C}, 5 \% \mathrm{CO}_{2}\right)$, tube numbers were counted under an IX71 inverted microscope.

\section{Xenograft tumors in vivo}


All experimental procedures involving animals were performed in accord with the "Guide for the Care and Use of Laboratory Animals" prepared by the National Academy of Sciences and published by the National Institutes of Health (NIH publication 86-23 revised 1985). Our animal investigations were performed in accordance with the institutional guidelines and were approved by the Animal Experimental Committee of The Sixth Affiliated Hospital of Guangzhou Medical University. Four-week-old male BALB/c nude mice were purchased from the Guangdong Experimental Animal Center of the Chinese Academy of Sciences and were bred and maintained in specific pathogen-free conditions. All mice were housed in a pathogenfree barrier facility with food and water ad libitum. Cells from each group were resuspended in serum-free DMEM at a density of $50,000,000$ cells per $\mathrm{ml}$ and then $0.1 \mathrm{ml}$ of the suspension was injected into the back of the nude mice. At the end of the treatment cycle $₫ 4$ weeks later》, all mice were euthanized by $\mathrm{CO}_{2}$ asphyxiation. Tumor tissues were obtained for CD31/periodic acid-Schiff (PAS) double staining.

\section{Survival analysis}

We performed Kaplan-Meir survival analysis on publicly available 'The Cancer Genome Atlas (TCGA)' data sets using 'Kaplan-Meir Plotter' database (https://kmplot.com/analysis/). The data of miR-138-5p was based on the miRNA subset. The HIF-1a and VEGFA mRNA cohort was based on the RNA-seq subset. We also split the dataset by auto select best cutoff in the system.

\section{Statistical analysis}

Data are shown on the ${ }^{\prime} \mathrm{c} \pm \mathrm{SD}$ manner, all data are gained by performed at least three repeats. Data werer analysis by SPSS 13.0 (SPSS, Inc.) and GraphPad Prism (GraphPad Software, Inc.). The chi-square test was used to examine the difference in miR-138-5p expression between paratumor and tumor tissues. Student's $t$-test or one-way ANOVA were used to assess the differences among groups. Statistical significance was set as below: * On behalf of $P<0.05$, ** On behalf of $P<0.01$, and $* \star \star$ On behalf of $P<$ 0.001 .

\section{Ethical considerations}

Our research has been approved by the The Sixth Affiliated Hospital of Guangzhou Medical University Ethics Committee (No. QPH-IRB-A0153). All patients involved in the study signed the informed consent. All experiments and public data acquisition were performed in accordance with ARRIVE guidelines and regulations.

\section{Results}

miR-138-5p is negative associated with VM densities, and can be used as the prognosis biomarker in HCC patients.

To determine the relationship between miR-138-5p expression and HCC, we conducted qRT-PCR to examine miR-138-5p expression levels. The median expression level was used as the cutoff. Low 
expression of miR-138-5p in 21 tissue (4 prar-tumor and 17 tumor tissue) was classified as values below the 50th percentile. High miR-138-5p expression in 25 tissue (19 para-tumor and 6 tumor tissue) was classified as values at or above the 50th percentile. As shown in Fig. 1A and 1B, the expression level of miR-138-5p in HCC tissues was lower than that in paratumor tissues. Similarly, miR-138-5p was expressed at lower levels in HCC cell lines than in the human normal hepatocyte cell line (MIHA) (Fig. 1C). Furthermore, we found that low expression of miR-138-5p was associated with a high VM density and high HIF-1 $a$ and VEGFA levels (Fig. 1D) and the expression of miR-138-5p was negative correlation with HIF-1a mRNA level (Fig. 1E and 1F). Furthermore, the survival results from Online Kaplan-Meier Plotter database indicated that low miR-138-5p, high HIF-1a and VEGFA mRNA levels were associated with a poor prognosis in HCC patients (Fig. 1G-I). Together, these data suggested that downregulation of miR138-5p was associated with a high VM density, high HIF-1 $a$ and VEGFA levels and might be associated with HCC progression.

Mir-138-5p Repressed Vm Formation By Hcc Cells

Our data shows the lower miR-138-5p level in HCC tissues indicated possible high VM density and poor prognosis. Biological significance in $\mathrm{HCC}$ progression and metastasis. We sought to determine whether miR-138-5p participates in the process of HCC VM. To this end, we conducted a tube formation assay to assess the effects of miR-138-5p knockdown/overexpression by a miR-138-5p inhibitor or miR-138-5p mimic. The expression levels of miR-138-5p after cell transfection with the miR-138-5p mimic or inhibitor are shown in Fig. 2A and 2B. Compared with the control group, $\mathrm{HCC}$ cells transfected with the miR-138-5p inhibitor exhibited a significantly enhanced VM formation ability, whereas this process was significantly repressed in HCC cells transfected with the miR-138-5p mimic (Fig. $2 \mathrm{C}$ and 2D). Our results suggested that the dysregulation of miR-138-5p expression in HCC cells might play an important role in HCC VM in vitro.

\section{Mir-138-5p Repressed Vessel Density In Vivo}

To determine the effects of miR-138-5p on HCC VM formation, we injected HepG2 cells that were treated with miR-138-5p inhibitor into the back of the nude mice. Mice injected with cells treated with miR-138-5p inhibitor had a higher VM density in the tumor tissue than the control group. (Fig. 3).

\section{miR-138-5p repressed the VM capacity by directly targeting HIF-1a in HCC cells}

HIF-1a is associated with VM ${ }^{[4,12]}$. Therefore, the effect of miR-138-5p downregulation on HIF-1a expression was investigated. The predicted structure of miR-138-5p from miRBase (http://mirtarbase.cuhk.edu.cn/) is shown in Fig. 4A. The TargetScan 3.1 online software predicted that HIF-1a was one of the putative target genes of miR-138-5p. The predicted result of binding site of miR138-5p with HIF-1a are shown in Fig. 4B. A luciferase reporter assay showed that HIF-1a was a direct target of miR-138-5p (Fig. 4C). As expected, transfection with the miR-138-5p mimic significantly reduced HIF-1 $\mathrm{a}$ and VEGFA mRNA and protein expression in HCC cells (Fig. 4D and 4F). Transfection with the miR138-5p inhibitor significantly enhanced HIF-1 $a$ and VEGFA mRNA and protein expression in HCC cells, and 
the HIF-1a inhibitor reversed the effects of the miR-138-5p inhibitor on HIF-1 $a$ and VEGFA mRNA and protein expression (Fig. 4E and 4F). The HIF-1a inhibitor CAY10585 also reversed the effects of the miR138-5p inhibitor on HCC cell tube formation (Fig. 4G). Therefore, we inferred that miR-138-5p influenced VM, at least partly, by regulating HIF-1a and VEGFA in HCC cells.

\section{Discussion}

Angiogenesis is the important hallmarks of tumor ${ }^{[13]}$. Tumor needs adequate blood supply to ensure enough nutrition for further development. Besides secreting angiogenic substances to induce endothelial cells to form tubes to produce more blood vessels ${ }^{[14]}$, tumor cells can formed new tube structure to add blood supply, this is called VM. VM is a recently described mechanism in which a blood supply is provided by tumor cells rather than endothelial cells, and it has been observed in certain highly aggressive tumors. MiRNAs, small noncoding RNAs of approximately 22 nucleotides in length, have been suggested to be important modulate factors in the process of VM in $\mathrm{HCC}^{[15]}$.

It is reported that a lower miR-138-5p level is associated with tumor progression and metastasis and that miR-138-5p can be considered to act as a tumor suppressor in most cancers ${ }^{[16-19]}$. In HCC research, recent studies have shown that miR-138-5p is frequently downregulated in HCC compared to controls and inhibits the occurrence and development of HCC. For example, Liu $Y$ et al. ${ }^{[20]}$ indicated that miR-138-5p could target and inhibit SOX9 expression and thus repress the cell proliferation and invasion in HCC. Syuan $L$ et al. ${ }^{[21]}$ found that miR-138-5p suppresses metastasis and tumorigenesis by enhancing vimentin expression and ubiquitination of cyclin E in HCC. However, the role of miR-138-5p in HCC VM has not been determined.

This study represents the first investigation of miR-138-5p in relation to VM in HCC. In this study, we found that miR-138-5p was downregulated in HCC tissues, and low miR-138-5p expression was significantly correlated with a poor prognosis of patients. Moreover, low miR-138-5p expression was related to a high EV density. Given the different basic tube formation ability in different cell lines. We choose the HepG2 and Hep3B cell line to carry out the gain or loss of function experiments according to the basic expression level of miR-138-5p and the basic tube formation ability. Consistently, in vitro experiments confirmed that miR-138-5p functioned as a tumor suppressor gene by inhibiting HCC VM.

miRNAs exert biological functions by regulating target genes by binding to their 3'-UTR. We found that HIF-1 a might be a target gene of miR-138-5p using bioinformatics tools. Using a dual-luciferase reporter assay, we demonstrated that miR-138-5p directly binds to the 3'-UTR of HIF-1 a mRNA and that miR-138$5 p$ expression is negatively related to HIF-1 a mRNA expression in HCC cell lines. HIF- $1 \mathrm{a}$ is a hypoxiaresponsive factor, which responses to hypoxia by activating as master regulator of the transcription of many genes, and participates in cell energy metabolism, angiogenesis, proliferation and apoptosis is. HIF$1 \mathrm{a}$ is frequently upregulated in many tumors. Studies have shown that HIF-1a acts as an oncogene and participates in tumor growth, metastasis ${ }^{[22]}$, metabolic rewiring and chemoresistance ${ }^{[23,24]}$. In addition, HIF-1a promotes tumor angiogenesis and VM and may be a prognostic biomarker for some tumors ${ }^{[25-}$ 
27]. Similarly, HIF-1 a promotes cell proliferation and migration in $\mathrm{HCC}^{[28]}$. In this study, we found that the miR-138-5p inhibitor enhanced HCC cell tube formation. Additionally, miR-138-5p targeted HIF-1a and downregulated its expression, and the effects of the miR-138-5p inhibitor on HCC cell tube formation were reversed by the HIF-1 a inhibitor CAY10585. All the results indicated that miR-138-5p suppressed VM in HCC by targeting HIF-1 $a$ and that HIF-1a was a medium for miR-138-5p in the process of HCC VM. Consistent with our results, Zhang JG, Wang M, and Roser Pinyol et al. found that HIF-1a played an important role in promoting $\mathrm{HCC} \mathrm{VM}^{[4,29,30]}$.

Increasing evidence demonstrates that VEGFA is the important downstream factors of HIF-1a. In this study, we found that when cells were transfected with the miR-138-5p mimic, HIF-1a and VEGFA expression was significantly reduced. Accumulating evidence has shown that VEGFA plays a key role in tumor angiogenesis and $\mathrm{VM}^{[12,31]}$. Therefore, miR-138-5p partly represses HCC VM by downregulating the expression of VEGFA. Considering the above findings, we demonstrated that miR-138-5p might act as a tumor suppressor by inhibiting VM via targeting HIF-1 $a$ and downregulating VEGFA and HIF-1a expression.

In conclusion, our study show that miR-138-5p was frequently downregulated in HCC tissues compared to paratumor tissues. Lower miR-138-5p expression was related to a high VM density, high VEGFA and HIF1a levels in HCC tissues. In addition, low miR-138-5p expression indicated a poor prognosis in HCC patients. In terms of the mechanism, miR-138-5p targets HIF-1a which is one of the upstream regulators of VEGFA, and HIF-1 $a$ and VEGFA participate in HCC VM. Thus, the current study links miR-138-5p, VEGFA, and HIF-1a to VM in HCC, and the role of miR-138-5p in HCC VM is clearly indicated. miR-138-5p may act as a potential therapeutic target in HCC.

\section{Abbreviations}

VEGFA

vascular endothelial growth factor $A$

qRT-PCR

quantitative real-time polymerase chain reaction

HCC

hepatocellular carcinoma

VM

vascular mimicry

OS

overall survival

$\mathrm{IHC}$

immunohistochemistry

HIF-1a

hypoxia-inducible factor $1 \mathrm{a}$. 


\section{Declarations}

\section{Acknowledgments:}

Thanks for the Department of General surgery, The Six Affiliated Hospital of Guangzhou Medical University, Qingyuan, China, for providing the HCC tissue samples and clinical data.

\section{Funding statement:}

This work was partly supported by grants from the Guangdong Medical Research Fund (No. 2018B133), the Qingyuan Science and Technology Projects (No. 2018A001) and the Qingyuan People's Hospital Fund (No. 20190213).

\section{Availability of data and material}

All data generated or analyzed during this study are included in this published article and its supplementary information files.

\section{Authors' contributions}

Hongwei Liu performed the experimental work and drafted the manuscript. Xiujin Hu, Weihe Tan, Peng Zhou, Yanmei Liu, Chen Chen, Meng Yang, Meifang Zhou, Shuxia Xuan, and Bing Cheng take part in the experiments. Weiguo Yin and Jinduan Lin conceived the study. All authors read and approved the final manuscript.

\section{Ethics and permissions}

The research protocol was approved by the Ethics Committee of The Six Affiliated Hospital of Guangzhou Medical University (No. QPH-IRB-A0153). All patients provided written informed consent. The authors confirm that all experiments and public data acquisition were performed in accordance with ARRIVE guidelines and regulations.

\section{Consent for publication}

All authors have read the manuscript and approved its submission to your journal.

\section{Competing interests}

The authors declare that there are no competing interests.

\section{References}

[1] Villanueva A. Hepatocellular Carcinoma[J]. N Engl J Med,2019,380(15):1450-1462. 
[2] Bray F, Ferlay J, Soerjomataram I, et al. Global cancer statistics 2018: GLOBOCAN estimates of incidence and mortality worldwide for 36 cancers in 185 countries[J]. CA Cancer J Clin,2018,68(6):394424.

[3] Xu J, Zhang Y, Wang Y, et al. Correlation of KAl1, CD133 and vasculogenic mimicry with the prediction of metastasis and prognosis in hepatocellular carcinoma[J]. Int J Clin Exp Pathol,2018,11(7):3638-3646.

[4] Wang M, Zhao X, Zhu D, et al. HIF-1alpha promoted vasculogenic mimicry formation in hepatocellular carcinoma through LOXL2 up-regulation in hypoxic tumor microenvironment[J]. J Exp Clin Cancer Res,2017,36(1):60.

[5] Kanchan R K, Siddiqui J A, Mahapatra S, et al. microRNAs Orchestrate Pathophysiology of Breast Cancer Brain Metastasis: Advances in Therapy[J]. Mol Cancer,2020,19(1):29.

[6] Yang H, Ma Q, Wang Y, et al. Clinical application of exosomes and circulating microRNAs in the diagnosis of pregnancy complications and foetal abnormalities[J]. J Transl Med,2020,18(1):32.

[7] Li B, Zhao H, Song J, et al. LINC00174 down-regulation decreases chemoresistance to temozolomide in human glioma cells by regulating miR-138-5p/SOX9 axis[J]. Hum Cell,2020,33(1):159-174.

[8] Wang Z, Yao Y J, Zheng F, et al. Mir-138-5p acts as a tumor suppressor by targeting pyruvate dehydrogenase kinase 1 in human retinoblastoma[J]. Eur Rev Med Pharmacol Sci,2017,21(24):56245629 .

[9] Wang X, Zhao Y, Cao W, et al. miR-138-5p acts as a tumor suppressor by targeting hTERT in human colorectal cancer[J]. Int J Clin Exp Pathol,2017,10(12):11516-11525.

[10] Zhang Y, Du X, Li W, et al. Resveratrol Improves Endothelial Progenitor Cell Function through miR-138 by Targeting Focal Adhesion Kinase (FAK) and Promotes Thrombus Resolution In Vivo[J]. Med Sci Monit,2018,24:951-960.

[11] Zhou W, Zhou W, Zeng Q, et al. MicroRNA-138 inhibits hypoxia-induced proliferation of endothelial progenitor cells via inhibition of HIF-1alpha-mediated MAPK and AKT signaling[J]. Exp Ther Med,2017,13(3):1017-1024.

[12] Wang H F, Wang S S, Zheng M, et al. Hypoxia promotes vasculogenic mimicry formation by vascular endothelial growth factor A mediating epithelial-mesenchymal transition in salivary adenoid cystic carcinoma[J]. Cell Prolif,2019,52(3):e12600.

[13] Hanahan D, Weinberg R A. Hallmarks of cancer: the next generation[J]. Cell,2011,144(5):646-674.

[14] Khorshidi A, Dhaliwal P, Yang B B. Noncoding RNAs in Tumor Angiogenesis[J]. Adv Exp Med Biol,2016,927:217-241. 
[15] Yang J, Lu Y, Lin Y Y, et al. Vascular mimicry formation is promoted by paracrine TGF-beta and SDF1 of cancer-associated fibroblasts and inhibited by miR-101 in hepatocellular carcinoma[J]. Cancer Lett,2016,383(1):18-27.

[16] Song N, Li P, Song P, et al. MicroRNA-138-5p Suppresses Non-small Cell Lung Cancer Cells by Targeting PD-L1/PD-1 to Regulate Tumor Microenvironment[J]. Front Cell Dev Biol,2020,8:540.

[17] Shi T T, Li R, Zhao L. Long noncoding RNA UCA1 regulates CCR7 expression to promote tongue squamous cell carcinoma progression by sponging miR-138-5p[J]. Neoplasma, 2020, 67, 1256-1265.

[18] Milanesi E, Dobre M, Bucuroiu A I, et al. miRNAs-Based Molecular Signature for KRAS Mutated and Wild Type Colorectal Cancer: An Explorative Study[J]. J Immunol Res,2020,2020:4927120.

[19] Zhang D, Liu X, Zhang Q, et al. miR-138-5p inhibits the malignant progression of prostate cancer by targeting FOXC1[J]. Cancer Cell Int,2020,20:297.

[20] Liu Y, Zhang W, Liu K, et al. miR-138 suppresses cell proliferation and invasion by inhibiting SOX9 in hepatocellular carcinoma[J]. Am J Transl Res,2016,8(5):2159-2168.

[21] Lin S L, Lin Y H, Chi H C, et al. A Novel Long Non-Coding RNA-01488 Suppressed Metastasis and Tumorigenesis by Inducing miRNAs That Reduce Vimentin Expression and Ubiquitination of Cyclin E[J]. Cells,2020,9(6).

[22] Zhao J, Xiao A, Liu C, et al. The HIF-1A/miR-17-5p/PDCD4 axis contributes to the tumor growth and metastasis of gastric cancer[J]. Signal Transduct Target Ther,2020,5(1):46.

[23] Khan H, Anshu A, Prasad A, et al. Metabolic Rewiring in Response to Biguanides Is Mediated by mROS/HIF-1a in Malignant Lymphocytes[J]. Cell Rep,2019,29(10):3009-3018.

[24] Tang J H, Ma Z X, Huang G H, et al. Downregulation of HIF-1a sensitizes U251 glioma cells to the temozolomide (TMZ) treatment[J]. Exp Cell Res,2016,343(2):148-158.

[25] Li W, Zong S, Shi Q, et al. Hypoxia-induced vasculogenic mimicry formation in human colorectal cancer cells: Involvement of HIF-1a, Claudin-4, and E-cadherin and Vimentin[J]. Sci Rep,2016,6:37534.

[26] Cai F F, Xu C, Pan X, et al. Prognostic value of plasma levels of HIF-1a and PGC-1a in breast cancer[J]. Oncotarget,2016,7(47):77793-77806.

[27] Cheng Z, Fu J, Liu G, et al. Angiogenesis in JAK2 V617F positive myeloproliferative neoplasms and ruxolitinib decrease VEGF, HIF-1 enesis in JAK2 V617F positive cells[J]. Leuk Lymphoma,2018,59(1):196203.

[28] Chen Y, Huang F, Deng L, et al. HIF-1-miR-219-SMC4 Regulatory Pathway Promoting Proliferation and Migration of HCC under Hypoxic Condition[J]. Biomed Res Int,2019,2019:8983704. 
[29] Zhang J G, Zhou H M, Zhang X, et al. Hypoxic induction of vasculogenic mimicry in hepatocellular carcinoma: role of HIF-1 alpha, RhoA/ROCK and Rac1/PAK signaling[J]. BMC Cancer,2020,20(1):32.

[30] Pinyol R, Montal R, Bassaganyas L, et al. Molecular predictors of prevention of recurrence in HCC with sorafenib as adjuvant treatment and prognostic factors in the phase 3 STORM trial[J]. Gut,2019,68(6):1065-1075.

[31] Liang $\mathrm{H}$, Xiao J, Zhou Z, et al. Hypoxia induces miR-153 through the IRE1 alpha-XBP1 pathway to fine tune the HIF1alpha/VEGFA axis in breast cancer angiogenesis[J]. Oncogene,2018,37(15):1961-1975.

\section{Figures}


A

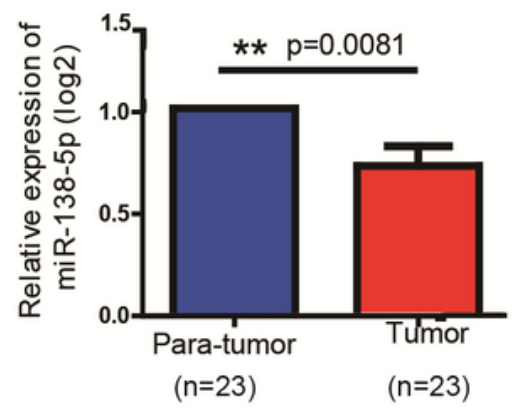

D
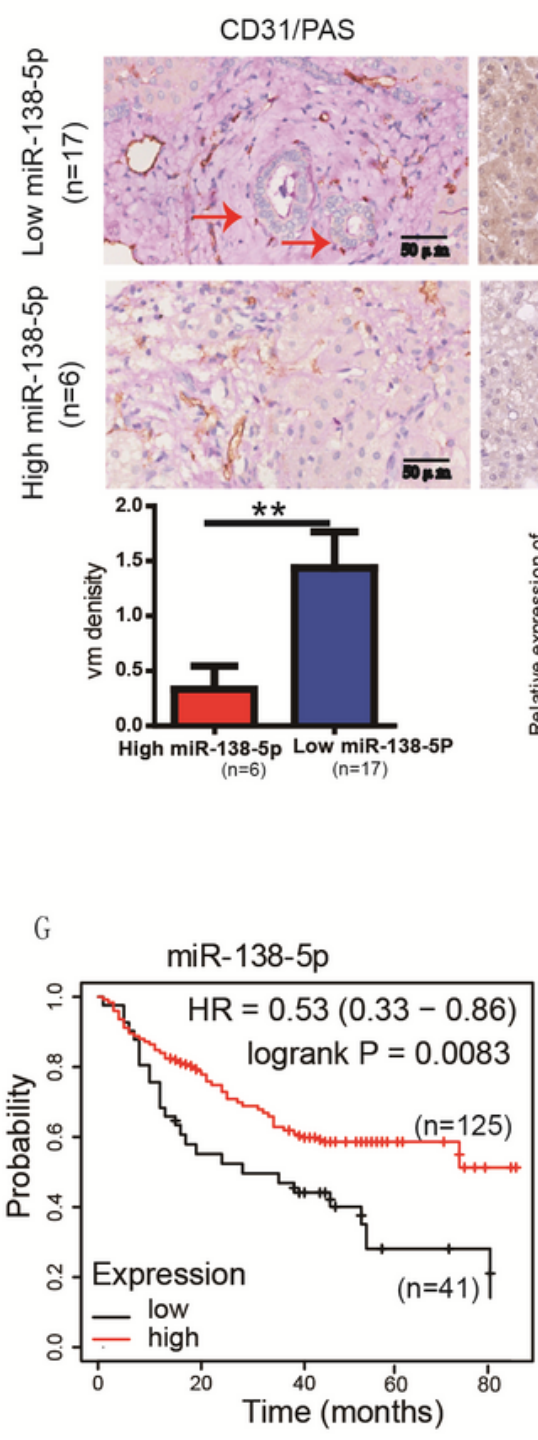

H

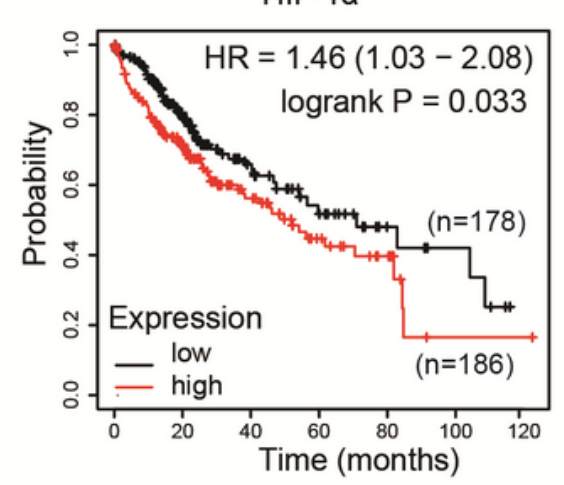

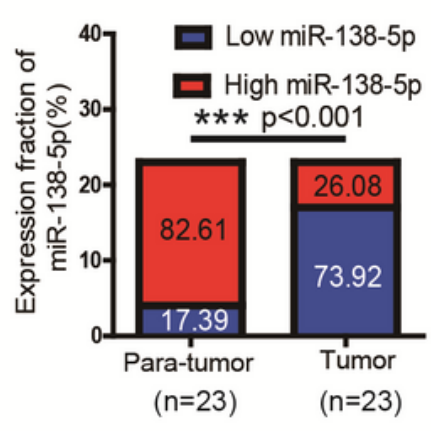

VEGFA
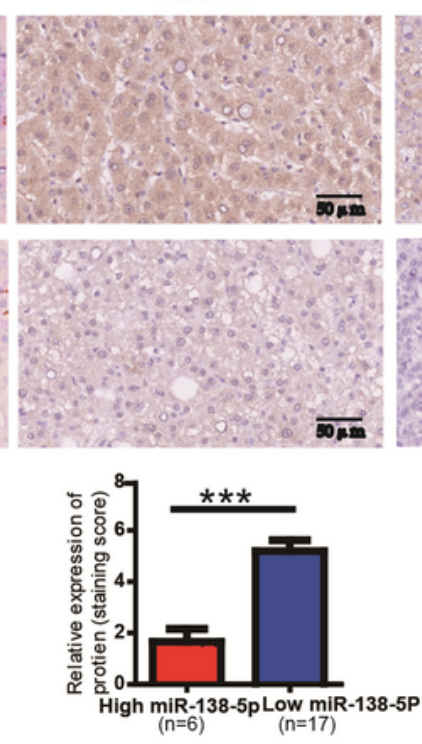

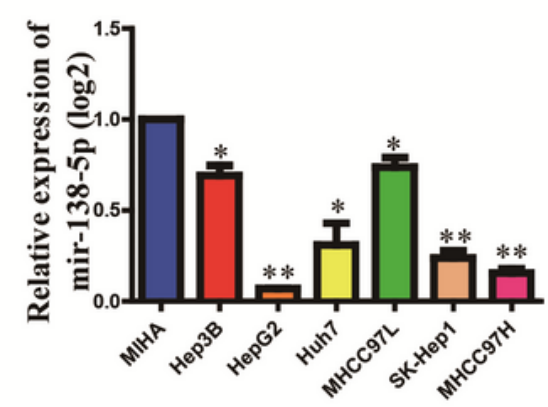

E

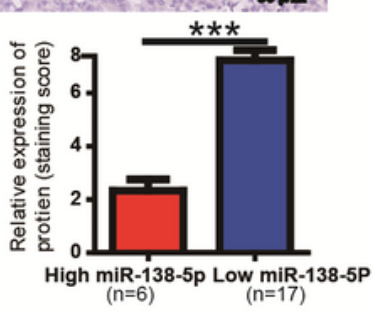

HIF-1a

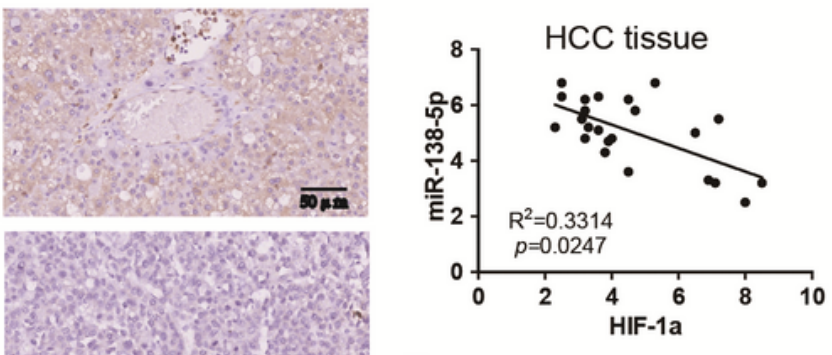

F

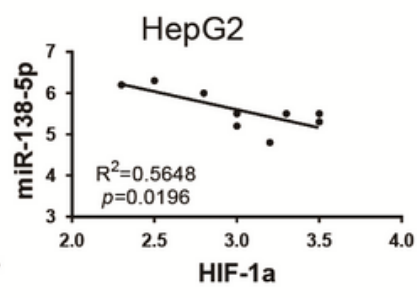

Figure 1

miR-138-5p is frequently downregulated in HCC tissues and HCC cell lines compared to controls, and low miR-138-5p expression is associated with a high VM density and high HIF-1a and VEGFA levels and indicates a poor prognosis in HCC patients. 
(A, B) RT-PCR analysis showed that miR-138-5p was more highly expressed in HCC tissues than in paratumor tissues. (C) RT-PCR analysis showed that miR-138-5p was more lower expressed in HCC cell lines than in human normal hepatocytes (MIHA cells). (D) CD31/PAS double staining was used to assess VM formation (red arrow). Immunohistochemical staining was used to assess HIF-1a and VEGFA expression. The results showed that miR-138-5p expression was related to the VM density and HIF-1a and VEGFA levels. (E) The correlation between miR-138-5p and HIF-1 a mRNA level in $23 \mathrm{HCC}$ tissues. The $\triangle \mathrm{Ct}$ values were subjected to Pearson correlation analysis. (F) The correlation between miR-138-5p and HIF1amRNA level in HepG2 cell line (9 repeats). The $\triangle \mathrm{Ct}$ values were subjected to Pearson correlation analysis. (G-I) The log-rank (Mantel-Cox) test showed that HCC patients with low miR-138-5p levels and high HIF-1a and VEGFA levels demonstrated worse OS than other patients from the KM-Plotter database.

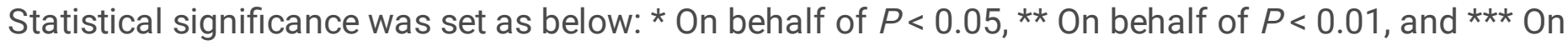
behalf of $P<0.001$.

\section{Figure 2}

\section{miR-138-5p reduces HepG2/Hep3B tumor cell tube formation.}

(A, B) Transfection efficiencies of the miR-138-5p mimic (A) and miR-138-5p inhibitor (B) in HCC cells. (C) Compared with the control, the miR-138-5p mimic reduced HepG2/Hep3B tumor cell tube formation. (D) The miR-138-5p inhibitor enhanced HepG2/Hep3B tumor cell tube formation. All experiments were repeated 3 times. Data are shown as the mean \pm SD. * On behalf of $P<0.05$.
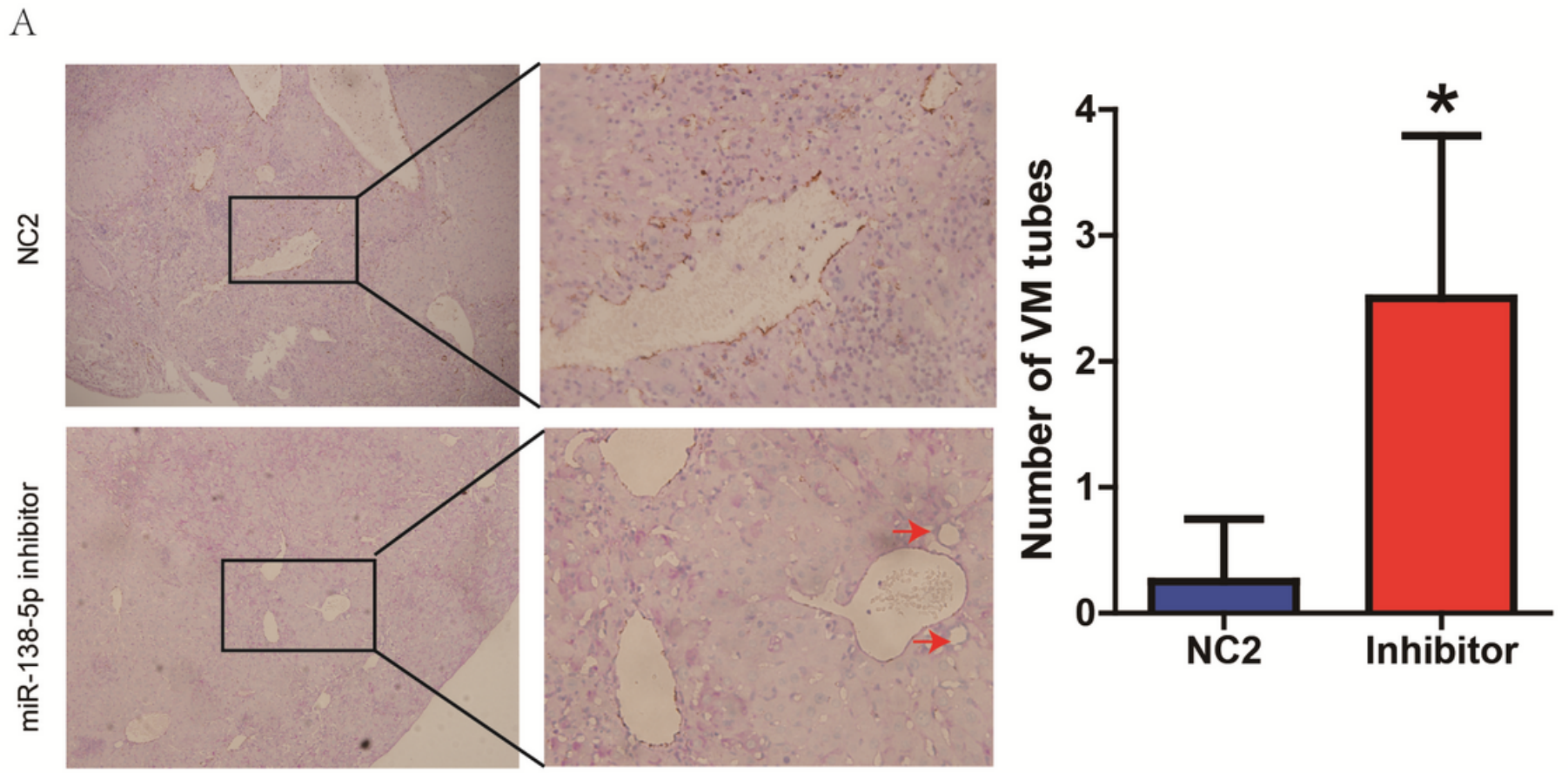

Figure 3 


\section{miR-138-5p repressed vessel density in vivo}

Nude mice results showed that when injected with HCC cells treated with miR-138-5p inhibitor, the density of VM was increased. Red arrow show the CD31(-)/PAS(+) vm structures. All experiments were repeated at 5 times. Data are shown as the mean \pm SD. * On behalf of $P<0.05$.

A

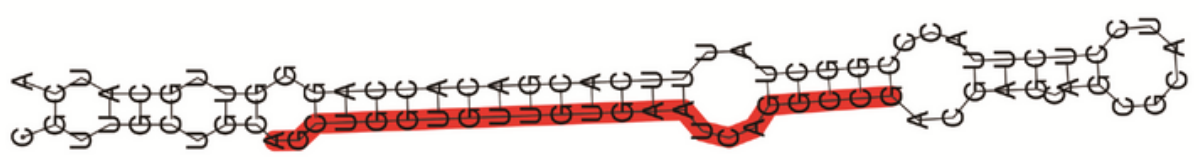

B

HIF-1 $\alpha-3$ ' UTR Wt 5 ' ... AUUUUCUUAAAAAAUACCAGCAG...3' |l|l| has-miR-138-5p 3' GCCGGaCUAagUGUUGUGGUCGA 5' HIF-1 $\alpha_{-}-3$ ' UTR Mut 5 ' ... AUUUUCUUAAAAAAUACCAGCAG...3'

C

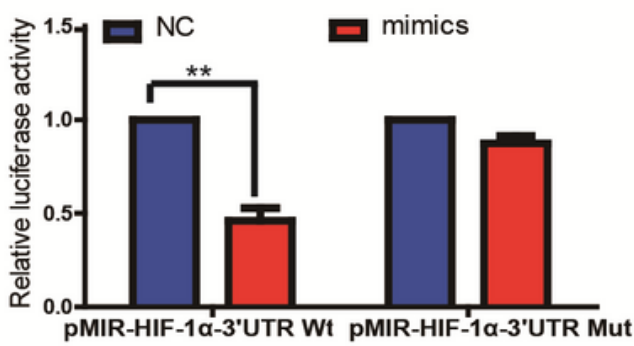

D

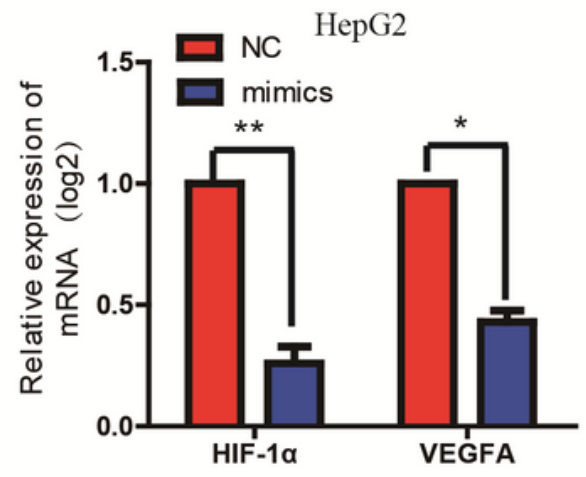

F

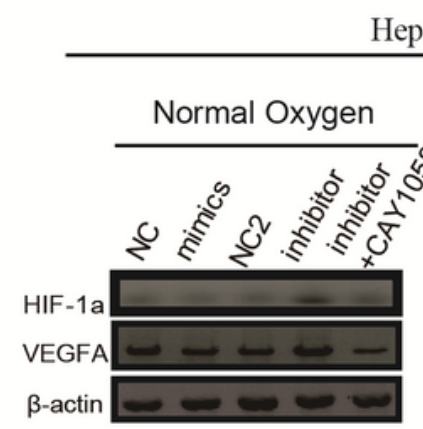

G

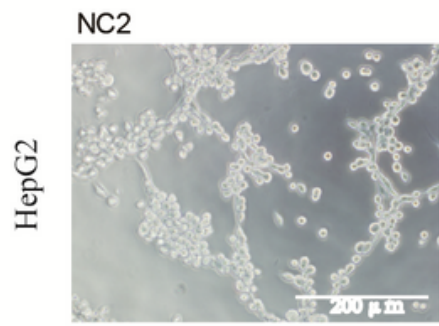

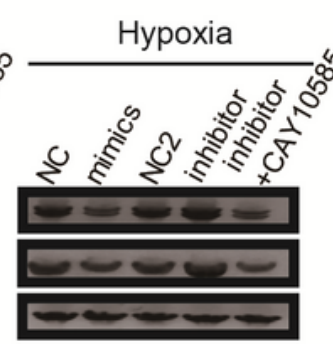

inhibitor
E
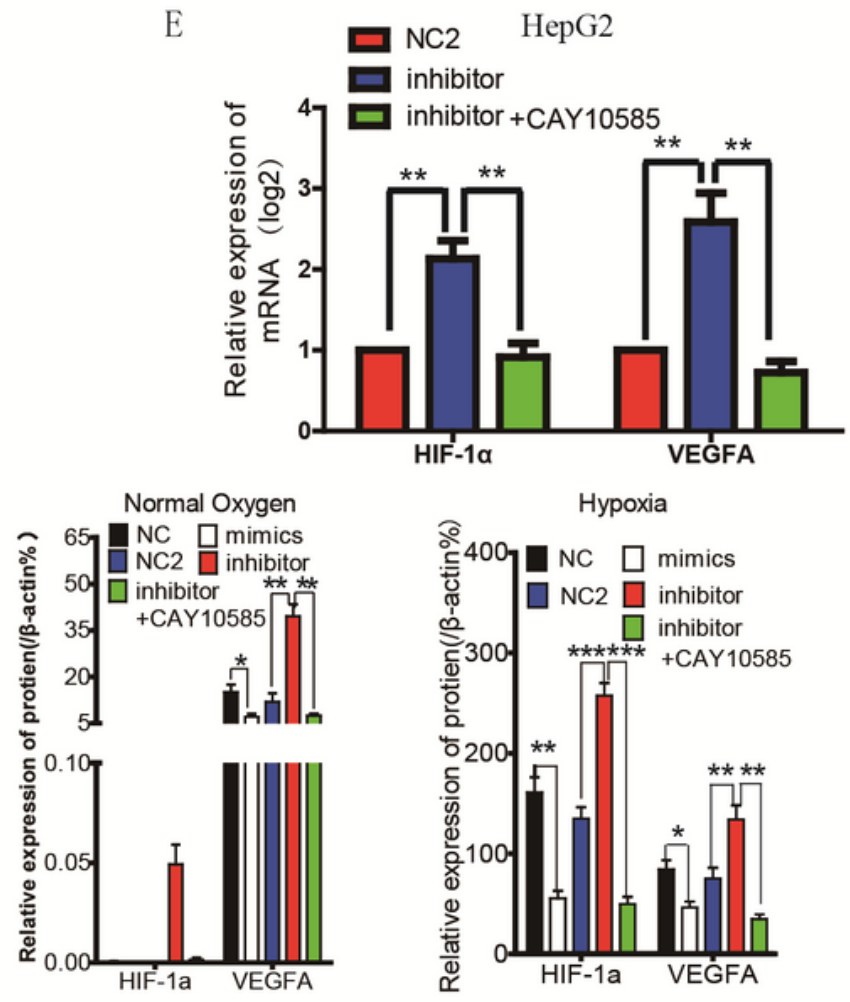

inhibitor+CAY10585
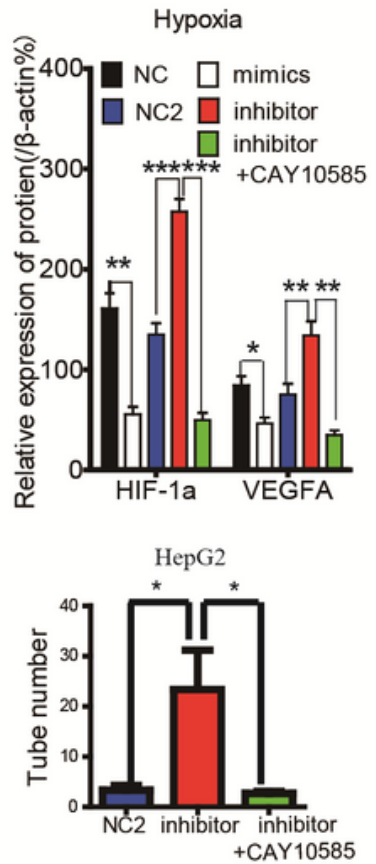

Figure 4 


\section{miR-138-5p reduces tumor cell tube formation by targeting HIF-1a/VEGFA signaling.}

(A) The structure of miR-138-5p was predicted from miRTarBase (http://mirtarbase.cuhk.edu.cn/). (B) The predicted binding site of miR-138-5p in the 3'-UTR of HIF-1a and the mutated 3'-UTR of HIF-1a. (C) A luciferase reporter plasmid containing the wild-type HIF-1 a 3'-UTR or the mutant HIF-1a 3'-UTR was transfected into 293T cells alone or cotransfected with the NC or miR-138-5p mimic, and luciferase activity was measured. (D, E) qRT-PCR detection of HIF-1a and VEGFA mRNA expression in HCC cells treated with the miR-138-5p mimic (D) or inhibitor (E). $(F)$ Western blot detection of HIF-1a and VEGFA protein expression in HCC cells transfected with the miR-138-5p mimic or inhibitor or with the HIF-1a inhibitor CAY10585 under normal oxygen and hypoxia conditions. (G) The HIF-1a inhibitor CAY10585 reversed the effects of the miR-138-5p inhibitor on tube formation by HCC cells. Data are shown as the mean \pm SD. All experiments were repeated at least 3 times. * On behalf of $P<0.05$; ** On behalf of $P<$ $0.01 ; * \star \star$ On behalf of $P<0.001$.

\section{Supplementary Files}

This is a list of supplementary files associated with this preprint. Click to download.

- Supplementtable1.docx 